\title{
Concomitant Cushing's Syndrome Due to Adrenal Adenoma in a Patient with Systemic Lupus Erythematosus
}

\author{
Masatoshi Shimizu, Masahito Kawata, Toshio OKadA*, Housai YuU*, Toshifumi Kurahashi**, \\ Kunito YAMANAKA** and Keiichi UMEZU**
}

\begin{abstract}
A 51-year-old woman had been administered $5 \mathrm{mg} /$ day of prednisolone due to systemic lupus erythematosus (SLE). She developed hypertension, hypokalemia and a pathologic pubic fracture during two years before admission. Although iatrogenic Cushing's syndrome was initially suspected, we diagnosed her as concomitant Cushing's syndrome due to a left adrenal tumor. The elevated endogeneous glucocorticoids were evaluated from urinary excretions of 17 hydroxycorticosteroids, which was 2 -fold higher than normal and equivalent to $10 \mathrm{mg}$ of prednisolone. After laparoscopic left adrenalectomy, SLE was favorably controlled with $15 \mathrm{mg}$ of prednisolone, the dosage of which was equivalent to the estimated amount of preoperative glucocorticoids. (Internal Medicine 41: 1044-1046, 2002)
\end{abstract}

Key words: prednisolone, autoimmune disease, hypokalemic hypertension, glucocorticoid, corticosterone

\section{Introduction}

Endogenous glucocorticoid excess could be obscured and erroneously interpreted as iatrogenic, if a patient is receiving steroids for a preceding autoimmune disease. Cushing's syndrome may be found unexpectedly during examinations for intractable hypertension (1). Following treatment of Cushing's syndrome, abrupt reduction of the endogenous glucocorticoids may induce a rebound in abnormal autoimmune activities (14). We present here a case of systemic lupus erythematosus (SLE) complicated by Cushing's syndrome. After the removal of an adrenal adenoma, SLE was favorably controlled with postoperative prednisolone equivalent to the estimated amount of preoperative glucocorticoids.

\section{Case Report}

A 51-year-old woman was referred to our division because of hypertension and fatigability in February 2000. She had been administered prednisolone for seven years due to SLE. At the age of 43 , she experienced dryness of the mouth and eyes. In the following year, she presented with malar rash, photosensitivity, arthritis and positive test for anti-DNA antibody. She was diagnosed as SLE and Sjögren's syndrome by a local physician, and steroid therapy was started. At the age of 49 , she became hypokalemic and hypertensive with a blood pressure of 156/92 mmHg. She was treated with a calcium antagonist. However, her blood pressure was gradually aggravated. In January 2000, she was admitted to our hospital because of a pathologic pubic fracture, which was managed conservatively for a month. She had been almost relieved from SLE with $5 \mathrm{mg} /$ day of prednisolone as a maintenance dose, but her blood pressure was poorly controlled despite treatment with nicardipine hydrochloride, alacepril and doxazosin mesilate.

On admission, the blood pressure was $198 / 106 \mathrm{mmHg}$ without difference between the two arms. Height was $159 \mathrm{~cm}$, and body weight was $37.2 \mathrm{~kg}$. The body mass index was $14.7 \mathrm{~kg} /$ $\mathrm{m}^{2}$, which remained unchanged throughout the clinical course. She lacked signs of Cushing's syndrome such as central obesity, buffalo hump or moon-face appearance. Complete blood cell count showed the following: erythrocyte $416 \times 10^{4} / \mathrm{mm}^{3}$, hemoglobin $12.2 \mathrm{~g} / \mathrm{dl}$, white cell count $10,400 / \mathrm{mm}^{3}$ with $8,450 /$ $\mathrm{mm}^{3}$ of neutrophils, and platelet count $27.0 \times 10^{4} / \mathrm{mm}^{3}$. Evaluation of serum chemistry revealed lactate dehydrogenase 674 $\mathrm{IU} / l$, alkaline phosphatase $879 \mathrm{IU} / l$, potassium $2.1 \mathrm{mEq} / l, \mathrm{C} 3$ $60 \mathrm{mg} / \mathrm{dl}$. C-reactive protein was negative and erythrocyte sedimentation rate was $12 \mathrm{~mm} / \mathrm{h}$. Anti-nuclear, anti-SS-A and antiSS-B antibodies were $\times 320$ (speckled type), $437.6 \mathrm{U} / \mathrm{ml}$ and $75.8 \mathrm{U} / \mathrm{ml}$, respectively. Assays for anti-DNA, anti-RNP, anti$\mathrm{Sm}$, anti-Scl-70 and anti-Jo-1 antibodies were all negative. Abdominal ultrasonography revealed a low echogenic mass in the left adrenal gland $30 \mathrm{~mm}$ in diameter (Fig. 1A). Abdominal computed tomography (Fig. 1B) and magnetic resonance im-

From the Division of Cardiology, *the Department of Internal Medicine and **the Department of Urology, Kobe National Hospital, Kobe Received for publication April 30, 2002; Accepted for publication July 4, 2002

Reprint requests should be addressed to Dr. Masatoshi Shimizu, the Division of Cardiology, Kobe National Hospital, 3-1-1 Nishiochiai, Suma-ku, Kobe 6540155 


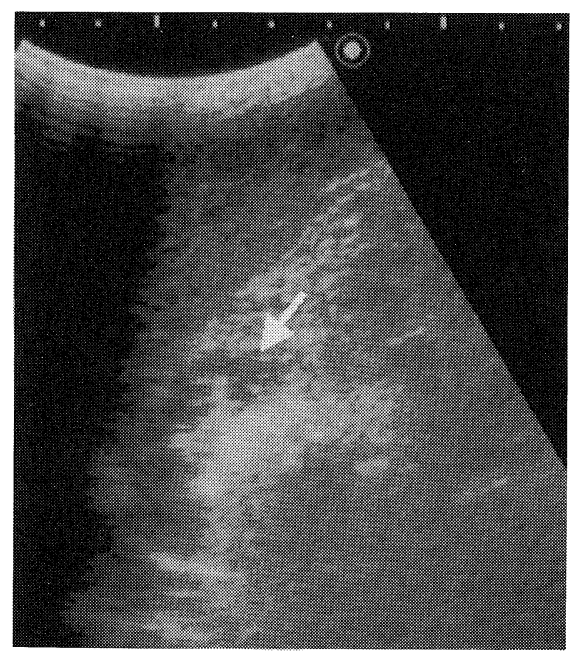

A

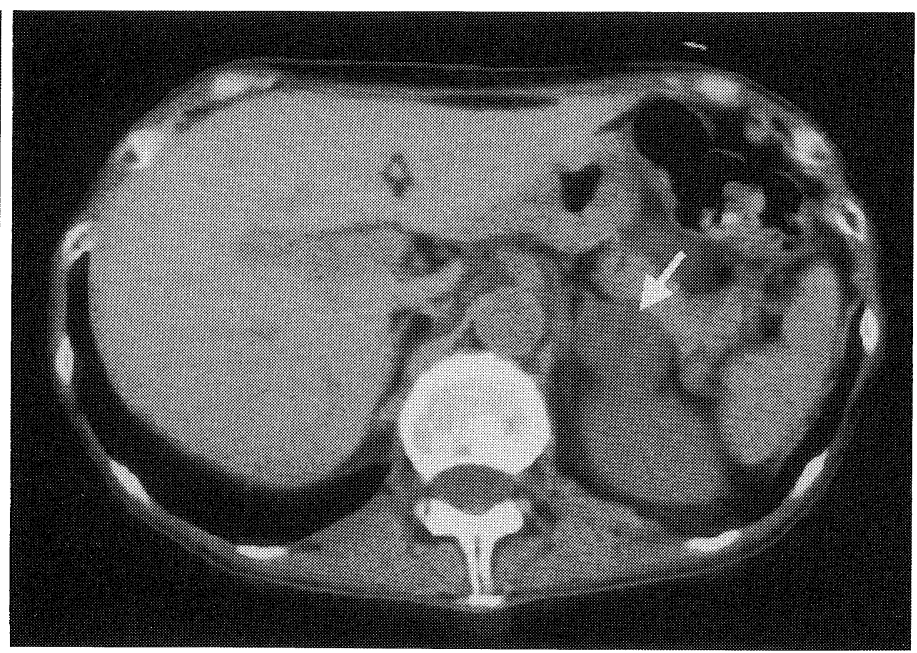

B

Figure 1. Abdominal ultrasonography (A) revealed a low echogenic mass in the left adrenal gland $30 \mathrm{~mm}$ in diameter (arrow). Abdominal computed tomography (B) also suggested a left adrenal tumor (arrow).

Table 1. Hormonal Evaluations

\begin{tabular}{|c|c|c|c|}
\hline \multicolumn{3}{|c|}{ Serum cortisol $(\mu \mathrm{g} / \mathrm{dl})$} & $(5.0-17.9)$ \\
\hline & 8:00 AM & 29.2 & \\
\hline & 3:00 PM & 30.6 & \\
\hline & 9:00 PM & 29.0 & \\
\hline \multirow[t]{2}{*}{ + dexamethasone } & $1 \mathrm{mg}$ & 20.6 & \\
\hline & $8 \mathrm{mg}$ & 24.2 & \\
\hline \multicolumn{2}{|c|}{ 24h urinary $17-\mathrm{OHCS}$ (mg/day) } & 15.7 & $(2.3-7.8)$ \\
\hline \multicolumn{2}{|c|}{ Serum corticosterone $(\mathrm{ng} / \mathrm{ml})$} & 17.3 & $(0.21-8.48)$ \\
\hline \multicolumn{2}{|c|}{ Plasma ACTH (pg/ml) } & $<4.0$ & $(4.4-48.0)$ \\
\hline \multicolumn{2}{|c|}{ DHEA-S (ng/ml) } & 90 & $(60-1,230)$ \\
\hline \multicolumn{2}{|c|}{ 24h urinary $17-\mathrm{KS}$ (mg/day) } & 6.80 & $(2.4-11.3)$ \\
\hline \multicolumn{2}{|c|}{ Plasma renin activity $(\mathrm{ng} / \mathrm{ml} / \mathrm{h})$} & 0.12 & $(0.2-2.7)$ \\
\hline \multicolumn{2}{|c|}{ Serum aldosterone $(\mathrm{pg} / \mathrm{ml})$} & 26.0 & $(56.9-150.3)$ \\
\hline
\end{tabular}

17-OHCS: 17-hydroxycorticosteroids, 17-KS: 17-ketosteroid, ACTH: adrenocorticotropin, DHEA-S: dehydroepiandrosterone sulfate.

aging also suggested a left adrenal tumor. We initially considered that her hypertension and hypokalemia could be due to long-term prednisolone therapy, but these findings prompted us to undertake further hormonal evaluations (Table 1). We obtained the following measurements after cessation of oral prednisolone for two days. Serum concentrations of cortisol determined at 8:00 AM, 3:00 PM, and 9:00 PM were 29.2, 30.6 , and $29.0 \mu \mathrm{g} / \mathrm{dl}$ (normal; $2.7-15.5$ ) respectively, which were constantly high without circardian changes. Twenty-four hour urinary excretion level of 17-hydroxycorticosteroids (17OHCS) was elevated to $15.7 \mathrm{mg} /$ day (normal; $2.3-7.8$ ). Serum corticosterone level was also elevated to $17.3 \mathrm{ng} / \mathrm{ml}$ (normal; $0.21-8.48$ ). Plasma adrenocorticotropin (ACTH) was undetectable. Plasma renin activity was $0.12 \mathrm{ng} / \mathrm{ml} / \mathrm{h}$ (normal; $0.2-2.7$ ), and serum aldosterone concentration was $26.0 \mathrm{pg} / \mathrm{ml}$ (normal; 56.9-150.3). Both of which were below the lower limit. Serum cortisol and urinary 17-OHCS excretion were not suppressed either by low dose ( $1 \mathrm{mg}$ for 2 days) or high dose ( $8 \mathrm{mg}$ for 2 days) of dexamethasone administration. Levels of other hormones were within normal range, including plasma dehydroepiandrosterone sulfate and urinary 17-ketosteroid. An adrenal scintigram showed exaggerated uptake of adosterol-I ${ }^{131}$ at the left adrenal gland. Based on these findings, we diagnosed her as concomitant Cushing's syndrome due to a left adrenal tumor.

The patient underwent laparoscopic left adrenalectomy in May 2000. A round adenoma $30 \times 25 \mathrm{~mm}$ in diameter was found at the left adrenal cortex. Microscopic examination revealed that the tumor consisted mainly of large clear cells. After surgery, she was administered $15 \mathrm{mg} /$ day of prednisolone as a replacement therapy for two months. She did not experience an exacerbation of SLE. Serum potassium level was elevated to $3.8 \mathrm{mEq} / l$, and her blood pressure declined to $138 / 88 \mathrm{mmHg}$ with doxazosin mesilate alone. Prednisolone was successfully tapered to $10 \mathrm{mg} /$ day. Two years after surgery, she remained well with relief of symptoms.

\section{Discussion}

Autoimmune disease is rarely complicated by Cushing's syndrome. There have been only five case reports in the literature including the present case (1-4). Notably, four of these five patients were Japanese females. The preceding autoimmune disease was SLE in two cases (1), and rheumatoid arthri- 
tis in the other three cases (2-4). The causes of subsequently developed Cushing's syndrome were due to adrenal adenoma in four and pituitary adenoma in one. When Cushing's syndrome was finally diagnosed, all of the patients were in a remissive state of their autoimmune disease. Two patients were under prednisolone treatment, one patient had been discontinued steroid therapy for eight years, and the remaining two patients had never received steroids. The symptoms related to concomitant Cushing's syndrome were hypertension, hypokalemia, pathologic fractures, and specific features such as central obesity and moon face. These conditions have played a clue to endogenous glucocorticoid excess in patients without steroid therapy. Iatrogenic Cushing's syndrome, however, was initially suspected in the three patients who had received steroid treatment for the preceding autoimmune disease. In the latter patients, adrenal tumors were unexpectedly found during examinations for intractable hypertension, or after an attempt to discontinue steroid therapy. In the present patient, hypokalemic hypertension and pathologic fractures were initially considered as iatrogenic, because she had been receiving long-term prednisolone treatment and lacked specific signs observed in Cushing's syndrome. Cushing's syndrome was diagnosed after we suspected hypokalemic hypertension of mineralocorticoid excess, and evaluated adrenal glands. We could not determine precisely when the present patient developed Cushing's syndrome, but we consider that the onset was two years before admission when she became hypertensive and hypokalemic.

The present case is the first report that the preceding autoimmune disease was favorably controlled after the treatment of concomitant Cushing's syndrome. In all four previous cases, a marked exacerbation of the preceding autoimmune disease was observed after removal of adrenal tumors or metyrapone treatment. These exacerbations occurred even when physiological dosage of hydrocortisone was administered subsequently. One patient with RA died of complicated pneumonia (2). Intensive prednisolone therapy was required in one patient with SLE (1), and the remaining two patients with RA needed methotrexate therapy $(3,4)$. Endogenous glucocorticoids have some anti-inflammatory effects. It is speculated that endogenous excess of glucocorticoids could have suppressed autoimmune activities. A rebound in abnormal autoimmune activities may be induced by abrupt reduction of the endogenous glucocorticoids following treatment of Cushing's syndrome. Physiological replacement with hydrocortisone may not be adequate to control disease activities. Therefore, it is reasonable to assess the elevated endogenous glucocorticoids and replace them with a sufficient dosage of prednisolone. We estimated endogeneous glucocorticoids from the 24 hour urinary excretion level of 17OHCS, which was 2-fold higher than normal and equivalent to $10 \mathrm{mg} /$ day of prednisolone (1). Adding the preoperative $5 \mathrm{mg} /$ day of prednisolone, we started $15 \mathrm{mg} /$ day of oral prednisolone postoperatively in the present patient. Because the relative anti-inflammatory effect of prednisolone is 4-fold stronger than cortisol, we considered that the dosage was sufficient to inhibit rebound excerabation of SLE.

Another unique characteristic of the present case is an elevated serum corticosterone level (5) with low plasma renin activity and low serum aldosterone concentration. Corticosterone, a precursor of aldosterone, is a weak mineralocorticoid, but is capable of causing hypokalemic hypertension. Corticosterone excess may explain low plasma renin activity and low serum aldosterone concentration in the present patient (6). Cushing's syndrome exceptionally demonstrates concurrent hypercortisolism and hypermineralocorticoidism $(7,8)$. We did not measure other mineralocorticoids such as deoxycorticosterone. Therefore, a possibility can not be throughly precluded that other undetermined mineralocorticoids had caused hypokalemic hypertension in the present patient. Although prednisolone dosage was adjusted to be equivalent to preoperative glucocorticoids, hypokalemic hypertension subsided after the removal of an adrenal adenoma.

\section{References}

1) Arima K, Higuchi M, Yoshizawa S, et al. Improvement of systemic lupus erythematosus activity by the association of delayed onset Cushing's syndrome. J Rheumatol 25: 2456-2458, 1998.

2) Nakamoto Y, Tabuchi Y, Saeki S, Mikami H, Ogihara T. Cushing's syndrome found during long-term glucocorticoid treatment for rheumatoid arthritis in an elderly woman. Jpn J Geriat 29: 54-58, 1992 (in Japanese, Abstruct in English).

3) Senécal JL, Uthman I, Beauregard H. Cushing's disease-induced remission of severe rheumatoid arthritis. Arthritis Rheum 37: 1826, 1994.

4) Yakushiji F, Kita M, Hiroi N, Ueshiba H, Monma I, Miyachi Y. Exacerbation of rheumatoid arthritis after removal of adrenal adenoma in Cushing's syndrome. Endocr J 42: 219-223, 1995.

5) Edwards OM, Galley JM, Tait AD, Mills IH. Steroid production rates in two patients with corticosterone producing adenomata. J Endocrinol 65: 52-53, 1975.

6) Yasuda G, Shionoiri H, Hayashi S, Umemura S, Ikeda Y, Ishii M. Cushing's disease: evaluation of mineralocorticoid -induced hypertension. Intern Med 32: 784-788, 1993.

7) Hogan MJ, Schambelan M, Biglieri EG. Concurrent hypercortisolism and hypermineralocorticoidism. Am J Med 62: 777-782, 1977.

8) Hobma S, Hermus A, Pieters G, Smals A, Kloppenborg P. Concurrent hypercortisolism and hyperaldosteronism due to an adrenal adenoma. Klin Wochenschr 68: 981-983, 1990. 